\title{
La Unidad de Pedagogía Universitaria y Didáctica del Derecho de la Facultad de Derecho de la Universidad de Chile: Su lugar en la historia de la reforma de la enseñanza del derecho
}

\author{
A Unidade Universitária de Pedagogia e Didática do Direito da Faculdade de Direito \\ da Universidade do Chile: Seu lugar na história da reforma do ensino do Direito
}

The Unit of University Pedagogy and Didactics of Law of the Faculty of Law of the University of Chile: Its place in the history of the reform of the teaching of Law

\section{María Francisca Elgueta-Rosas \\ Universidad de Chile}

RESUMEN Transcurridos trece años desde la formación de la Unidad de Pedagogía Universitaria y Didáctica del Derecho, parece necesario realizar un primer ejercicio de posicionamiento de su lugar en la historia de la reforma de la enseñanza del derecho en Chile y en Iberoamérica. La unidad surgió en el contexto de un nuevo momento histórico del interés por promover la reforma de la enseñanza universitaria (por ende, del derecho) y ha hecho una contribución para la formación de una comunidad académica que se propone fortalecer la pedagogía jurídica como espacio de investigación.

PALABRAS CLAVE Unidad de Pedagogía Universitaria y Didáctica del Derecho, pedagogía jurídica, congresos de pedagogía universitaria y didáctica del derecho, aprendizaje del derecho, reforma de la enseñanza del derecho.

RESUMO Treze anos após a formação da Unidade Universitária de Pedagogia e Didática do Direito, parece necessário realizar um primeiro exercício para posicionar o seu lugar na história da reforma do ensino do Direito no Chile e na América Latina. A Unidade surge no contexto de um novo momento histórico de interesse na promoção da reforma do ensino universitário (daí do Direito) e tem dado um contributo para a formação de uma comunidade académica que pretende fortalecer a Pedagogia J. 
jurídica, congressos de pedagogia universitária e didática do direito, aprendizagem do direito, reforma do ensino do direito.urídica como espaço de investigação.

ABSTRACT Thirteen years after the formation of the Unit of University Pedagogy and Didactics of Law, it seems necessary to carry out a first exercise to position its place in the history of the reform of the teaching of Law in Chile and Latin America. The unit emerged in the context of a new historical moment of interest in promoting the reform of university education (hence of Law) and has made a contribution to the formation of an academic community that intends to strengthen legal pedagogy as a space for research.

KEYWORDS University Pedagogy and Didactics of Law Unit, legal pedagogy, congresses of university pedagogy and didactics of law, law learning, reform of law teaching.

\section{Un poco de historia: Inspiración de la Unidad de Pedagogía Universitaria y Didáctica del Derecho}

La Unidad de Pedagogía Universitaria y Didáctica del Derecho (UPUDD) se siente heredera de la reflexión de Aníbal Bascuñán que ya en el año 1954, en su obra Pedagogía jurídica, soñaba con que se fundara una unidad especializada en el aprendizaje del derecho al interior de las escuelas de derecho latinoamericanas, inspirado en los trabajos de Valentín Letelier (1852-1919), Adolfo Posada (1860-1944), Rafael Altamira y Crevea (1866-1951) y Juan Antonio Iribarren (1885-1977), quienes venían denunciando, desde fines del siglo XIX, las limitaciones que presentaba la enseñanza positivista del derecho, el abuso de la memorización del texto legal y de los manuales (Elgueta y Palma, 2018).

Desde un punto de vista temporal, la Unidad retoma el trabajo realizado por el Instituto de Docencia e Investigación Jurídica, entidad ocupada de la reforma de la enseñanza, que agrupó desde 1969 hasta 1975 a las cinco facultades de derecho chilenas de la época: Universidad de Concepción, Universidad de Chile, Pontificia Universidad Católica de Chile, Universidad Católica de Valparaíso y Universidad de Valparaíso. Surgió gracias al financiamiento de la Fundación Ford y de la Universidad de Standford. Su herencia más sustantiva fue el Boletín de Docencia e Investigación Jurídica, que aportó de manera sistemática a una reflexión multidisciplinar a través de artículos, transcripción de congresos y seminarios, trabajos y conferencias de expertos nacionales e internacionales a la enseñanza del derecho, sociología jurídica e investigación jurídica (Benfeld, 2016).

En sus seis años de existencia, el boletín publicó 29 números, en los que de manera clara y comprometida se fortalece una reflexión en torno a la pedagogía jurídica. En la iniciativa participaron no solo profesores de derecho, sino también pedagogos y 
sociólogos. El primer número, señero en marcar una línea de trabajo, promueve la innovación curricular y de mejora de la enseñanza del derecho. Contiene los resultados de la Convención para la Reforma de los Estudios de Derecho de la Universidad de Chile, junto con un artículo de André Tunic en el que propone «salir del Neolítico» (Tunic, 1969). Todos estos documentos están encaminados a promover el cambio y la reforma en la enseñanza del derecho. Este primer número anuncia el rol que asume el instituto: promover innovación y cambios en las aulas de derecho.

El Boletín difundió, con apoyo de la Fundación Ford, las investigaciones y reflexiones de la comunidad de profesores que viajaron a Estados Unidos para formarse en metodologías activas, promoviendo un semillero que modernizara una desactualizada formación jurídica (Merryman, 2000). Llegó a su fin en 1975 cuando terminó el aporte económico de Estados Unidos y ninguna autoridad universitaria o estatal chilena consideró relevante su proyección.

Como consecuencia de ello, entre 1975 y 2007 la temática de la enseñanza del derecho no fue trabajada sistemáticamente en las escuelas de derecho chilenas: solo se reforzó la tradicional clase magistral meramente expositiva (Elgueta y Palma, 2014), con una marcada formación positivista y memorística.

En 2008, en el contexto de la masificación de la educación superior promovida por los gobiernos de la Concertación de Partidos por la Democracia (1990-2010) y la Nueva Mayoría (2014-2018), nace la UPUDD (UPUDD, 2018). Acompañó al fenómeno la promoción de la innovación en la educación superior a través de los proyectos de Mejoramiento de la Calidad y Equidad en la Educación Superior (Mecesup) que, financiados con un préstamo del Banco Mundial, promovían «la implementación inicial [...] de una nueva arquitectura curricular para la educación superior basada en resultados de aprendizaje y demostración de competencias. [...] La definición e implementación inicial de un sistema para la transferencia de créditos académicos (SCT-Chile, compatible con el ECTS europeo). [...] La consolidación del mejoramiento de la calidad de la oferta educacional terciaria y su innovación permanente, en concordancia con la planificación estratégica de las IES y las resoluciones de la Comisión Nacional de Acreditación (CNA, Ley 20.129)». ${ }^{1}$

En este nuevo contexto, que promueve en la educación superior un fortalecimiento de la arquitectura curricular, de la enseñanza-aprendizaje, de desarrollo de competencias y de cuenta pública a través de mediciones estandarizadas de los resultados de los proyectos, la Unidad de Pedagogía Universitaria y Didáctica del Derecho comienza a promover la formación y la reflexión en torno a la pedagogía jurídica.

Otro aspecto que facilitó el auge de la pedagogía jurídica, que también obedece al contexto nacional, es el desarrollo de los procesos de acreditación institucional y de

1. Más información en la presentación de la Universidad de Chile, disponible en https://bit. ly/3JysiNa. 
carreras que, entre otros factores, miden la calidad de la docencia. Además, en 2007 se inició un movimiento estudiantil, con una manifestación fuerte en 2011, que demandó políticas públicas en pos de una educación de calidad y gratuita (Palma, 2014; y Palma y Elgueta, 2019).

Por lo tanto, desde sus inicios la UPUDD tuvo claridad acerca de que había un entorno nacional e internacional favorable para los cambios, y que la dificultad se encontraba en el contexto interno: la resistencia cultural de las facultades de derecho. Por lo mismo, tuvo que salir al encuentro de los expertos nacionales y extranjeros que, aislados, cultivaban la pedagogía jurídica.

\section{Acciones en favor de la reforma del aprendizaje del derecho}

En este entorno, con un contexto nacional propicio, la UPUDD ha venido desarrollando acciones para fortalecer a la pedagogía jurídica como disciplina, considerando necesario modernizar las prácticas para fortalecer los procesos de enseñanza y aprendizaje, así como la innovación y reflexión curricular en la carrera de Derecho; y no ha descuidado realizar aportes al momento de generar políticas públicas en educación superior. En estos trece años ha organizado congresos nacionales e internacionales; ${ }^{2}$ ha realizado actividades de docencia ${ }^{3}$ en pre $^{4}$ y posgrado, ${ }^{5}$ hasta concretar un diplomado en la materia; ${ }^{6}$ ha diseñado y ejecutado investigaciones de campo de tipo exploratorias (Elgueta-Rosas, Zamorano y Palma, 2015; Sanhueza, Elgueta-Rosas y Palma, 2018; Elgueta-Rosas, Silvestre y Spaolinzi, 2019; Elgueta-Rosas, 2016a; Elgueta-Rosas, 2016b; Elgueta-Rosas, Palma y Lunelli, 2016; Elgueta-Rosas, Zamorano y Palma, 2019; Elgueta-Rosas e Infante, 2016; Elgueta-Rosas y Palma, 2015); ha desarrollado acciones de perfeccionamiento de profesores y ayudantes; ${ }^{7} \mathrm{y}$ acciones de acompañamiento a estudiantes para fortalecer sus aprendizajes. Resultados más concretos son la creación de la cátedra de Pedagogía Jurídica en el pregrado y del postgrado el Diploma en Pedagogía Jurídica, sostenidos sobre la base de un aprendizaje activo e investigación educativa. La Unidad también fundó la Revista de Pedagogía Universitaria y Didáctica del Derecho y ha publicado libros y artículos.

Todo el trabajo a lo largo de estos años se ha ido manifestando en el crecimiento paulatino de la Revista Pedagogía Universitaria y Didáctica del Derecho. Siguiendo los lineamientos de los tiempos presentes, la revista ha colaborado dando presencia a la

2. https://www.facebook.com/watch/?v=155255291741094.

3. http://www.derecho.uchile.cl/noticias/139499/

juristas-chilenos-y-brasilenos-analizan-desafios-pedagogicos.

4. https://www.facebook.com/100057397700329/videos/361255298058293.

5. https://www.facebook.com/watch/?v=2863680060437417.

6. https://postgrados.derecho.uchile.cl/diploma-en-pedagogia-juridica/.

7. http://www.derecho.uchile.cl/noticias/138851/

upudd-impulsa-ciclo-de-conversaciones-sobre-educacion-superior. 
voz de la comunidad de expertos que viene bregando en la última década por cambiar la cultura tradicional de las escuelas de derecho.

Los congresos nacionales e internacionales han permitido el diálogo entre los que hasta ese momento eran profesores aislados y excepcionales. En 2010 se realizó el Primer Congreso Nacional de Pedagogía Universitaria y Didáctica del Derecho. El evento académico contó con la asistencia de especialistas en pedagogía universitaria y procesos de aseguramiento de la calidad, junto con una naciente comunidad académica proveniente de escuelas de derecho públicas y privadas de todo el país que tímidamente llegó a compartir sus experiencias docentes: ha sido paulatino el paso desde una comunidad que comparte experiencias de innovación docente a una que desarrolla investigación en pedagogía jurídica. Ya tenemos a nuestro haber once congresos, ${ }^{8}$ nacionales e internacionales.

El primer congreso internacional se celebró en la Facultad de Derecho de la Universidad de Chile, en 2012, ${ }^{9}$ con un amplio programa: ${ }^{10}$ el segundo se realizó en el Instituto de Investigaciones Jurídicas de la Universidad Nacional Autónoma de México, en 2014; ${ }^{11}$ el tercero se celebró en la Facultad de Derecho de la Universidad de Buenos Aires, en 2016; el cuarto se celebró en Brasil, en la Universidad Federal de Rio Grande do Sud en conjunto con la Universidad del Ministerio Público, ${ }^{12}$ en 2018; el quinto se realizó en $2019^{13} \mathrm{y}$ el sexto congreso en $2021,{ }^{14}$ ambos de manera virtual. Todos los congresos han contado con un amplio auspicio de instituciones internacionales, lo que da cuenta de un proceso de internacionalización y generación de redes.

El alto número de especialistas que a lo largo de estos trece años han fomentado

8. https://www.youtube.com/watch?v=jUYmdjZKnSU, https://www.facebook.com/ watch/?v=1970923726543637, http://www.derecho.uchile.cl/noticias/125967/congreso-de-pedagogiadel-derecho-analizo-ley-de-educacion-superior, http://www.derecho.uchile.cl/noticias/137723/miradas-en-torno-a-los-procesos-de-formacion-de-los-futuros-abogados, http://www.derecho.uchile.cl/ noticias/87141/consolidan-red-de-pedagogia-universitaria-y-didactica-del-derecho, http://www.derecho.uchile.cl/noticias/77009/debaten-sobre-pedagogia-universitaria-en-segundo-congreso-del-area-.

9. http://www.derecho.uchile.cl/noticias/68526/ afianzan-lazos-con-instituto-de-investigaciones-juridicas-de-la-unam.

10. file://C:/Users/Maria\%2oFrancisca/Downloads/programa-congreso-internacionalpedagoga-ydidctica2012\%20(1).pdf.

11. http://www.derecho.uchile.cl/noticias/107007/ facultad-promueve-acciones-en-pedagogia-y-didactica-del-derecho.

12. https://www.facebook.com/watch/?v=2269621519717134, https://www.facebook.com/ watch/?v=1989927921300653.

13. http://www.derecho.uchile.cl/noticias/181998/amplia-convocatoria-en-vi-congreso-intlde-pedagogia-universitaria, http://www.derecho.uchile.cl/noticias/169700/academicos-deamerica-africa-y-europa-participan-en-el-cpudd, http://www.derecho.uchile.cl/noticias/148084/ acuerdan-formacion-de-la-asociacion-chilena-de-ensenanza-del-derecho,

14. https://www.youtube.com/watch?v=Jw1UGK-dybQ. 
el desarrollo de la disciplina de la Pedagogía Jurídica ha sido la base del nacimiento y fortalecimiento de la Revista Pedagogía Universitaria y Didáctica del Derecho. El énfasis del trabajo de la UPUDD ha estado en el desarrollo de una comunidad de académicos que investigan, publican y reflexionan en torno a los temas propios de esta disciplina.

Hoy se puede afirmar:

- Que existe una comunidad académica en formación y constante crecimiento ocupada de reflexionar los temas propios de la pedagogía jurídica.

- Que se viene desarrollando investigación de campo, cuantitativa y cualitativa, además de la bibliográfica, con una mirada de diálogo internacional y transdisciplinaria.

- Que a los congresos y seminarios impulsados por la UPUDD están convergiendo otros especialistas y otras redes, de diversas latitudes y bajo distintos paradigmas.

Que tenemos como desafío formar a nuevas generaciones sin perder de vista el legado de los viejos maestros de fines del siglo XIX y mediados del siglo XX.

\section{Referencias}

BENFELd Escobar, Johann (2016). «La discusión sobre la enseñanza del derecho en chile dentro del nuevo paradigma universitario: Una tarea pendiente». Revista de derecho (Coquimbo), 23 (1): 143-171. Disponible en https://www.scielo.cl/scielo. php?script=sci_arttext\&pid=So718-97532016000100007\&lng=en\&nrm=iso\&tlng =en.

Boletín de Docencia e Investigación JuRídica (2021). Todos los números se pueden consultar acá: http://drevistas.ucv.cl/numero_por_titulo.php?tituloID=60.

Elgueta-Rosas, María Francisca (editora) (2012). «Actas Primer Congreso Nacional de Pedagogía Universitaria y Didáctica del Derecho». Facultad de Derecho, Universidad de Chile.

-. (2016a). Prueba Codice-Derecho: Una experiencia de evaluación en la educación superior. Santiago: Universidad de Chile.

-. (2016b). «Hacia una concepción de práctica de docencia en la formación profesional». En Cecilia Lorea, Octavio Van-Dúnen y Ana Clara Correa, Contemporaneidade, imagens da justiça e ensino jurídico. Vol. 1. Pesquisa e práticas inovadoras no ensino jurídico contemporâneo (pp. 199-220). Brasil: Casa Leiria.

Elgueta-Rosas, María Francisca, Eric Eduardo Palma y Francisco Zamorano Figueroa (2015). «Primer estudio nacional de caracterización de estudiantes de Derecho». Unidad de Pedagogía Universitaria y Didáctica del Derecho, Facultad de Derecho, Universidad de Chile. 
-. (2019). «Percepciones y valores de jóvenes y estudiantes de Derecho (1994-2018) como antecedentes del proceso formativo en derechos humanos». En Ricardo Rabinovich (editor), Los derechos humanos desde la historia. Buenos Aires: Universidad de Buenos Aires.

Elgueta-Rosas, María Francisca, Ana Carolina Farías Silvestre y Ana Elisa Spaolonzi (editoras) (2019). Pedagogía jurídica: Contribuciones y reflexiones latinoamericanas. Santiago: Ril.

Elgueta-Rosas, María Francisca, Eric Palma e Isabella Cristina Lunelli (2016). "Conhecimento, iconografia e ensino do direito". En Coleção imagens da justiça. Vol. 2. Pesquisa e práticas inovadoras no ensino jurídico contemporâneo. São Leopoldo: Casa Leiria.

Elgueta-Rosas, María Francisca y Sanndy Infante (2016). «Implicancias e impacto de la prueba Codice derecho en la Universidad de Chile: Alfabetización académica en vistas al género jurídico». En Eliane Lousada, Anise Ferreira, Luzia Bueno, Roxane Rojo, Solange Aranha y Lília Abreu-Tardelli, Diálogos brasileiros no estudo de gêneros textuais/discursivos (pp. 1.202-1.213). Araraquara: Letraria.

Elgueta-Rosas, María Francisca y Eric Palma (2014). «Una propuesta de clasificación de la clase magistral impartida en la Facultad de Derecho». Revista Chilena de Derecho 41 (3): 907-924. DOI: http://dx.doi.org/10.4067/So718-34372014000300006

-. (2018). «Enseñanza de la Historia del Derecho centrada en el aprendizaje de los estudiantes a lo largo de 115 años de la fundación de la cátedra». Precedente, 12: 29-62. DOI: https://doi.org/10.18046/prec.v12.2649.

Merryman, John (200o). «Law and development memoirs I: The Chile Law program», The American Journal of Comparative Law, 48 (3): 481-499.

Palma, Eric (2014). En defensa de la educación pública, del derecho a la educación y de la legalidad universitaria. Santiago: Orión.

Palma, Eric y María Francisca Elgueta-Rosas (2019). Aprendizaje y didáctica del derecho. Ciudad de México: Tirant lo Blanch.

Sanhueza, José Miguel, María Francisca Elgueta-Rosas y Eric Eduardo Palma (2018). «Masificación, segmentación y satisfacción en estudiantes de Educación Superior en Chile: El caso de la carrera de Derecho». Revista Espacios, 39 (52): 27-47.

Tunic, André (1969). «Salir del Neolítico». Boletín de docencia e investigación jurídica. Unidad de Pedagogía Universitaria y Didáctica del Derecho. Memoria Institucional (2008-2018). Prólogo de Rogelio Pérez Perdomo. Disponible en https://www.ericeduardopalma.cl/publicaciones-y-documentos/95-libro-memoria-de-la-unidad-de-pedagogi-a-2008-2018). 


\section{Sobre la autora}

María Francisca Elgueta-Rosas es doctora en Educación, Universidad de Valladolid. Diplomada en Docencia Universitaria, Universidad Metropolitana de Ciencias de la Educación. Diplomada en Gestión Universitaria, Universidad Metropolitana de Ciencias de la Educación. Diplomada en Diseño e Implementación de Encuestas, Pontificia Universidad Católica de Chile. Investigadora Unidad de Pedagogía Universitaria y Didáctica del Derecho, Facultad de Derecho, Universidad de Chile. Su correo electrónico es cpudd2@derecho.uchile.cl. (D) https://orcid.org/oooo-0002-4212-3960. 
La Revista Pedagogía Universitaria y Didáctica del Derecho (RPUDD) es una publicación científica semestral que contribuye a la reflexión multidisciplinaria sobre pedagogía universitaria y didáctica del derecho, para la formación y consolidación de esta área de investigación; así como a la difusión de prácticas innovadoras en la enseñanza-aprendizaje del derecho considerando el contexto nacional e internacional. Es una publicación electrónica internacional con una codirección entre Brasil y Chile.

\author{
DIRECTORA \\ María Francisca Elgueta Rosas \\ Universidad de Chile \\ DIRECTOR \\ Renato Duro Dias \\ Universidad Federal de Rio Grande, Brasil \\ SITIO WEB \\ pedagogiaderecho.uchile.cl \\ CORREO ELECTRÓNICO \\ rpedagogia@derecho.uchile.cl \\ LICENCIA DE ESTE ARTÍCULO
}

Creative Commons Atribución Compartir Igual 4.o Internacional

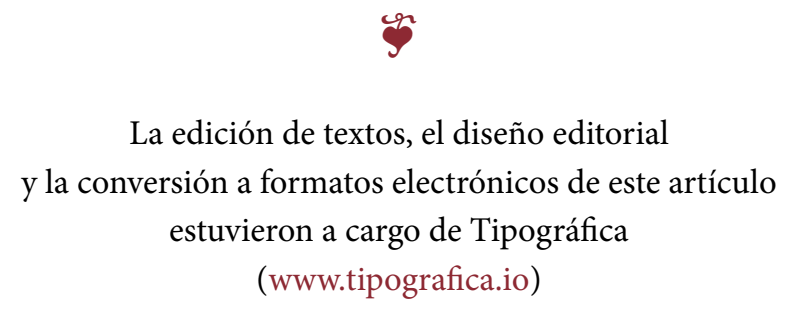

